\author{
Marie-Hélène MASSOT \\ Directeur de Recherche, \\ Laboratoire Ville, Mobilité, Transport \\ Institut National de Recherche sur les Transports et leur Sécurité \\ Champs-sur-Marne
}

\author{
Jean-Pierre ORFEUIL \\ Professeur \\ Directeur du Laboratoire C.R.E.T.E.I.L. \\ Université Paris XII - Créteil
}

\title{
La mobilité au quotidien, entre choix individuel et production sociale
}

\section{Résumé}

On a longtemps rendu compte des comportements de déplacements des hommes dans l'espace par un terme renvoyant à une logique collective et de masse, celui de migration (résidentielle, quotidienne). L'intégration dans l'observation et la compréhension de toute la palette des motifs de déplacements et l'individualisation croissante des pratiques ont amené l'usage d'un terme plus générique, emprunté aux sciences sociales et notamment à ceux qui s'intéressent à la fluidité dans l'espace social, celui de mobilité. Ce glissement n'est pas purement sémantique : il exprime le passage de l'empire de la nécessité aux capacités stratégiques et aux désirs des acteurs, de la représentation aréolaire d'un territoire rassemblant une "société locale" "contenant" les déplacements de ses membres à des représentations réticulaires (le monde réseau, les villes globales), multi-territoriales (les sociétés d'archipel).

Sur tous ces points, les enquêtes initiées dans les années soixante par les ingénieurs pour alimenter leurs modèles de trafic nous ont permis, grâce à l'élaboration d'indicateurs, de concepts, de représentations nouvelles, de comprendre en profondeur la dynamique et les rôles respectifs des rationalités individuelles et de la formation de normes sociales dans le champ spécifique de la mobilité quotidienne. C'est de cette construction dont nous rendons compte dans cet article, qui livre aussi à partir des quelques résultats saillants des recherches conduites, les convictions fortes en matière de dynamique de la mobilité qui s'en dégagent et les paradigmes qu'il conviendrait de mieux explorer.

\begin{abstract}
Spatial behaviour was studied in the past through group concepts, such as residential or regular migrations from home to work. The will to understand travel behaviour for the full range of trip purposes and the growing personalisation of behaviour leads to adopt the term of mobility from social sciences. This change is not purely semantic. It expresses the transition from a vision based of the needs to the consideration of the strategic capabilities of the persons, from areola-based representations of a local society to network and archipelago-based representations of individual behaviours.

We have used, in conjunction with the international community of travel behaviour analysts, the travel surveys introduced in the sixties to feed traffic models, to build concepts, indicators and representations of travel behaviour, to understand in depth the transformations of mobility
\end{abstract}


patterns and to analyse the roles of individual rationality and social norms. The paper relates this history, highlights some key results and strong beliefs and questions the need to explore new paradigms.

Mots clé : mobilité quotidienne, représentations, indicateurs, comportements, dynamique. Keywords : daily mobility, concepts, indicators, behaviour, dynamics.

\title{
La mobilité au quotidien, entre choix individuel et production sociale
}

\author{
"La caractéristique la plus remarquable des sociétés occidentales \\ contemporaines est bien leur grande mobilité"
}

SOROKIN P.A.

1927

\section{Introduction}

La mobilité occupe une place centrale dans les pratiques, les attentes et les imaginaires contemporains, et sur une palette si diverse de registres qu'elle devient fédératrice d'un sentiment de changement de société. En témoignent par exemple le goût des jeunes pour les " villes qui bougent ", l'explosion des prothèses nomades et des " solutions mobilité " de l'industrie des NTIC, la croissance d'un tourisme international qui rend toute destination accessible au moins acculturé, le rêve éveillé d'un marché du travail plus flexible par de nombreux employeurs, la flexibilité des personnalités d'individus " hors sol ", ou d"' individus trajectoires" (Ehrenberg A., 1995), engagés dans des relations éphémères (Aubert N., 2004), etc. Il y a bien là le signe d'une société où les cartes de l'ordre sont rebattues à vive allure, et de l'approfondissement d'un "procès de personnalisation en rupture avec la phase inaugurale des sociétés modernes, démocratiquedisciplinaire, universaliste-rigoriste" (Lipovetski G., 1983). Cette présence de la mobilité dans des domaines aussi divers suggère aussi l'existence d'interdépendances multiples entre les différentes formes de mobilité, et invite à une lecture globale des évolutions, comme celle de "modernité liquide" avancée par Bauman (Bauman Z., 2000). Elle ne doit en revanche pas condamner, à notre sens, toute approche sectorielle, sous l'accusation de réductionnisme. En effet, de même que le concept de marché n'a pas épuisé la diversité des formes de marchés encastrées dans des relations sociales et des mécanismes institutionnels très divers, de même les formes concrètes de la production de la mobilité varient fortement d'un champ à l'autre. Un examen rapide, champ par champ, montre l'existence d'évolutions de rythme différent, voire de sens opposé : on sait depuis longtemps que les informations et certains capitaux circulent mieux que les biens, qui circulent eux-mêmes mieux que les hommes ; on a redécouvert avec Castel (Castel R., 1995) que la construction d'un lien fort entre employeurs et employés était une construction sociale récente, et que la mobilité de la main d'œuvre était probablement plus forte au dix-neuvième siècle qu'aujourd'hui. Le développement de la propriété du logement, la persistance d'un système résidentiel local familial (Bonvalet C., 1999), la montée des phénomènes qualifiés de Nimby, 
comme celle des ségrégations socio-spatiales, l'humilité des politiques devenus très attentifs à la "proximité" suggèrent a minima des schèmes où l'insertion territoriale conserve quelque pertinence...

Comprendre en profondeur la dynamique d'un champ, les rôles respectifs des rationalités individuelles et de la formation de normes sociales, baliser les perspectives de ce champ suppose donc un investissement spécifique. C'est de cet investissement, démarré il y a une vingtaine d'années, sur les déplacements des hommes sur les territoires du quotidien, dont nous rendons compte ici. Ce champ avait été exploré depuis une trentaine d'années par des ingénieurs, des géographes, des économistes, des urbanistes qui, au-delà de leurs divergences, partagent une même éthique scientifique : un même souci d'allers retours entre leurs constructions théoriques et les observations, de recherche des points de repère condition de toute objectivité (Durkheim E., 1894) ; une même conviction que les comportements reposent sur des fondations, pour reprendre les termes de Castel, qu'il leur appartient de mettre à jour ; un même credo enfin, celui qu'il y a encore un sens à se placer du point de vue de l'ensemble, à identifier des mécanismes suffisamment généraux influant de façon suffisamment nette les comportements de groupes de personnes.

Leurs apports spécifiques ont été essentiels dans la compréhension de tel ou tel aspect de cette question complexe qu'est la formation et la dynamique de la mobilité, et c'est la raison pour laquelle nous en rendons compte dans une première partie. La focalisation de ces apports sur des points particuliers, la ligne de partage assez étanche entre ceux qui se plaçaient à un niveau microsocial et ceux qui se placent à un niveau macrosocial, la culture fondamentalement "transport" de la plupart d'entre eux rendaient toutefois difficile l'élaboration d'une vision globale des dynamiques, qui passe nécessairement par la prise en compte des interactions entre les systèmes de transport, les systèmes d'interaction sociale, et les systèmes de localisations. Il faut pour cela construire des hypothèses, des concepts, en bref une manière de voir, qui soit de plus opérationnalisable à travers la construction d'indicateurs. C'est de cette construction dont nous rendons compte dans une seconde partie. Nous exposons enfin dans une troisième partie quelques résultats saillants des recherches conduites, les convictions fortes en matière de dynamique de la mobilité qui s'en dégagent et les paradigmes qu'il conviendrait de mieux explorer.

\section{Penser la mobilité des hommes dans l'espace : des apports multiples}

La compréhension des interactions des hommes dans l'espace a longtemps mobilisé les géographes, plutôt sur le champ interurbain où le coût de l'interaction était le plus élevé. Ils en ont tiré des représentations de l'intensité des échanges (les modèles gravitaires où l'interaction entre deux villes dépend des potentiels de chacune d'elle, des populations, des productions, selon la nature de la question) et de la viscosité (résistance opposée par l'espace au mouvement) et des lois sur les aires de marché de chacune des places et les distances entre villes. Avec la croissance urbaine, celle de la motorisation et des congestions des réseaux, les administrations des transports ont été amenées à se poser des problèmes de même nature à l'échelle intra-métropolitaine : quels niveaux d'échanges, aujourd'hui et plus encore demain, entre quartiers d'une même aire métropolitaine ? C'est Alan Voorhes (1959) qui proposera, aux Etats-Unis, des modèles de prévision à long terme des niveaux de demande entre zones : les distributions des populations et des activités, supposées exogènes, planifiées et / ou prévisibles et les niveaux de motorisation résultant de la croissance économique déterminent des niveaux de demande et permettent de programmer les réseaux susceptibles de la satisfaire. L'approche est certes agrégée dans son 
principe, mais sa mise en œuvre suppose la réalisation d'enquêtes (longtemps appelées origine / destination) retraçant les pratiques de déplacements des individus ${ }^{1}$. La méthode sera utilisée un peu partout dans le monde par des administrations, surtout routières, engagées dans des investissements lourds pour lesquels un propos très agrégé sur les flux, les migrations alternantes et leur caractère prédéterminé étaient suffisants, ce qui explique sans doute que la communauté sociologique se soit, pour l'essentiel, tenue à l'écart de l'analyse d'enquêtes perçues comme très techniques. Les enquêtes qui servent de base aux ajustements nécessaires aux prévisions constitueront pourtant progressivement un corpus international sur les pratiques spatiales que des géographes, des économistes, des socio-économistes ne tarderont pas à s'approprier pour de toutes autres fins que la prévision mécaniste à long terme des trafics, des investissements et des conditions de circulation. Les économistes (dont Mac Fadden David, futur prix Nobel) développent à l'époque des modèles de choix discrets sur une base d'individualisme méthodologique très poussée. On leur doit une analyse désagrégée de la valeur (révélée, ou déclarée) des attributs des déplacements (valeur du temps, du confort, des aménités annexes, voire des destinations) d'une nature comparable à celles qui sont développées dans le domaine de la valorisation des biens immobiliers (méthodes hédoniques, consentement à payer) qui est un premier pas vers l'individuation des analyses des pratiques de déplacements. Un second pas est franchi par Hägerstrand T. (1970) et sa géographie du temps (1987), qui cherche à représenter ce que peuvent faire les individus en fonction de la distribution des activités sur le territoire, mais aussi de leur situation propre, ou même des changements dans leur situation: dotation en moyens de transport, rôles familiaux et sociaux générateurs de contraintes spatio-temporelles et aptitudes à lever partiellement ces contraintes par chaînage de déplacements, regroupement d'activités ou encore reports d'activités sur une autre personne ou lors d'une autre sortie. Il sera bientôt suivi par des français, des anglais, des allemands ${ }^{2}$ qui, utilisant les enquêtes standard ou construisant leurs propres bases d'informations originales, se retrouveront sous la bannière de l'"analyse par les activités" et emprunteront aux sociologues le terme de mobilité utilisé depuis Sorokin (1927) pour la mobilité sociale. Ils substituent aux flux, migrations alternantes, prévisions à long terme, les notions de mobilité, d'arbitrages, de stratégies complexes. Ils étudient la " mobilité de la mobilité ", c'est-à-dire les transformations des routines et leurs liens avec les changements familiaux, professionnels ou autres. C'est en particulier à Goodwin (Goodwin P. et Kitamura R., 1989) que l'on doit des analyses fines du changement de pratiques : statistiquement, les pratiques de mobilité changent fortement car il y a toujours une fraction de la population en changement dans les sphères professionnelles, familiales ou autres. En revanche, lorsque la situation de l'individu est stable, de nouvelles opportunités ne sont pas toujours perçues du fait d'inerties, de seuils de perception ou encore de nécessité de rechercher l'information. Ils participent ainsi, dans l'étude du rapport de l'homme à l'espace et au temps, à l'élaboration d'une vision individuée et

\footnotetext{
${ }^{1}$ Ces enquêtes présentent indiscutablement une dimension fonctionnaliste : elles renseignent les motifs, les lieux de départ et d'arrivée, les moments, les durées et les distances, les moyens de transport empruntés. Elles ne disent en revanche rien sur les attentes qu'on pouvait avoir, les conditions de déplacement, l'importance attachée au déplacement, le plaisir ou le désagrément qu'on a retiré à se déplacer.. ;

2 Plusieurs dizaines d'enquêtes nationales, plusieurs centaines d'enquêtes dans des zones métropolitaines, de nombreux exercices comparatifs internationaux (Salomon et coll, 1993, Newman et coll., 1989, Kenworthy et coll., 1998, Shafer et coll., 2000). Malgré certaines faiblesses identifiées depuis longtemps, ce corpus, mettant en rapport des individus socialement et spatialement situés et des pratiques, satisfait aux conditions énoncées par de Vulpian (de Vulpian, 2004) pour identifier les dynamiques porteuses : " présenter un caractère international et être nourries par un système amont vigoureux ".
} 
postmoderne des phénomènes, tenant compte toutefois de l'encastrement des projets de mobilité dans les réseaux de relations familiales et sociales. On ne peut pourtant pas, surtout lorsqu'on s'intéresse aux dynamiques des phénomènes, s'en tenir à des analyses micro-sociales. Chaque décision individuelle participe à moyen et long terme à la formation d'effets de club (un système de transport collectif fonctionne mal avec peu d'usagers, le marché de l'occasion participe à la diffusion du produit automobile) et d'effets de congestion. Le politique est alors interpellé, et chaque changement dans les conditions de la mobilité (amélioration des vitesses, proportion plus grande de la population équipée de moyens de transport) s'accompagne de réactions systémiques (maillage des services, stratégies de localisation des acteurs), qui à leur tour changent les conditions de la mobilité, etc.. On peut tenter de rendre compte de ces phénomènes par des modèles complexes traitant simultanément de l'occupation de l'espace et des conditions de la mobilité. Cependant le mérite revient à Y. Zahavi (1974), inspiré par les travaux de Szalai (1972) sur les usages du temps, d'avoir proposé une vision simple et stratégique de la dynamique de la co-production de l'espace et des conditions de la mobilité, vision réfutable au sens de Popper. Il postule que les citadins cherchent à maximiser les opportunités que l'espace leur offre sous une double contrainte, celle d'une limite dans le temps de déplacement quotidien et celle d'une limite dans la part du budget monétaire allouées à ces déplacements, cette dernière étant toutefois variable selon que les personnes sont ou non équipées d'automobiles. Les enquêtes dont il dispose suggèrent que ces limites sont en moyenne de l'ordre d'1h à $1 \mathrm{~h} 30$ selon la taille de la ville, et de $5 \%$ à $10 \%$ du budget selon que les ménages sont non motorisés ou motorisés: de meilleures conditions de mobilité (sous forme de vitesse, de baisse des coûts ou d'accès croissant à l'automobile) permettent aux individus d'enrichir leur programme d'activités, ou d'étendre leur territoire, ou d'éloigner leur " base résidentielle " des lieux d'activités, dans un espace-temps défini par certaines contraintes. La vision est présentée en termes individualistes car c'est ainsi qu'elle peut être validée ou invalidée par les enquêtes menées dans la population, mais elle peut aussi bien relever d'une approche plus macroscopique dont nous rendrons compte infra. C'est pourquoi notre projet de compréhension des dynamiques de la mobilité articule des dimensions micro sociales et des dimensions macro sociales, des dimensions de court terme et des dimensions de long terme.

\section{Rénover la compréhension des pratiques de mobilité quotidienne : paradigmes et indicateurs}

C'est sur la combinaison de ces bases que nous avons construit des concepts, des indicateurs et des catégories d'analyses susceptibles de rendre compte des pratiques de déplacements et de leurs évolutions (Orfeuil JP., 2000) à court et à long terme et plus précisément que ne le font les simples recueils statistiques (Certu, 2002) publiés en routine. Il faut d'abord caractériser au mieux, avec l'information disponible, les pratiques. Le déplacement pris isolément est un vecteur d'information intéressant, mais ne constitue pas en soi une unité d'analyse pertinente. L'unité pertinente est une " tranche de vie " (la semaine ou le mois pour certaines enquêtes spécifiques, la journée pour la plupart des autres) qui permet de rendre compte des pratiques spatiales, sociotemporelles et des modes d'organisation. C'est donc au niveau d'une période que sont construits les principaux indicateurs de mobilité, non seulement le nombre de déplacements par type d'activités qui les motivent, la structure des enchaînements de ces déplacements et des moyens de transport utilisés, les parties du territoire fréquentées mais aussi l'ensemble des coûts de cette mobilité pour l'individu (coût en temps, monétaire). Cette mise en ordre du quotidien de l'individu débouche notamment sur la constitution de " budgets " : budgets-temps de 
déplacements (BTT) somme de toutes les durées de déplacements, budget monétaire, budgetdistances, ensemble des distances parcourues par jour, révélateur de la capacité à connecter des territoires éloignés, etc.. La mobilité individuelle ne se résume toutefois pas à la seule impulsion individuelle à se déplacer, et nous avons postulé, en nous éloignant de la vision " individualiste " de Zahavi, qu'elle est aussi (et surtout) une production sociale. Les contraintes budgétaires mises en avant par l'auteur ne s'appliquent en effet qu'en moyenne à la population de référence de la ville, ce qui n'implique évidemment pas que tous les individus satisfassent individuellement la contrainte. Notre interprétation propose une vision fondée sur une propriété de réflexivité du système urbain et du système de déplacements. Les entreprises et les services optimisent leur stratégie spatiale (localisations des établissements, maillage) en prenant en compte les durées et les coûts d'accès dans les moyens de transport les plus répandus, qui définissent alors l'étendue et les caractéristiques sociales des bassins de recrutement et de chalandise. Cette étendue varie en fonction des conditions de la mobilité (degré de motorisation des populations, vitesses praticables sur les réseaux, coûts, etc.). Ce qu'on appelle improprement la "loi des budgets temps constants" n'implique donc a priori aucun parti pris en faveur de l'individualisme méthodologique. C'est une représentation stylisée d'un processus réflexif de co-construction des lieux et des liens. Les pratiques de mobilité sont alors co-produites par un ensemble de facteurs économiques, organisationnels et culturels. Il importe dès lors de suivre, notamment dans une perspective dynamique, les lieux d'habitat et d'activités, les rythmes qui leur sont propres et les usages et rythmes découlant des rôles familiaux et sociaux des individus.

Les principaux indicateurs et catégories d'analyses découlent d'une "opérationnalisation" de ces deux perspectives et relèvent de cinq dimensions : (i) La première est celle de l'interaction sociale : les besoins individuels de co-présence hors du domicile sont qualifiés par le nombre de déplacements par jour, le nombre de sorties du domicile, le temps passé hors du domicile, le nombre d'activité réalisées en dehors du domicile, désagrégés en catégories plus ou moins grandes et qualifiantes (activités ou motifs plus ou moins contraintes par le corps social). (ii) La seconde est celle de l'interaction spatiale qui rend compte de l'intensité spatiale et des types de territoires ou espaces connectés par les individus. La taille de l'espace fréquenté est appréhendée par la distance totale parcourue par jour. La structure des connexions territoriales l'est par grands types de liaisons (interne à Ville-centre, à la banlieue et aux espaces périurbains, échanges entre la ville centre-la banlieue, la ville centre et le périurbain, ou entre la banlieue et les espaces périurbains mais aussi échanges entre territoires plus lointains). Notre souci a été ici d'inscrire au plus large les mobilités individuelles en permettant à chacun, par construction analytique, de dépasser les territoires administratifs des enquêtes locales et en travaillant sur les interactions territoriales les plus lointaines (mise en relation entre centres villes de deux agglomérations, entre tissus urbains les plus reculés de deux agglomérations..). L'analyse des territoires des individus, soit l'ensemble des lieux qu'il fréquente quotidiennement, plus difficile à mener, ne relève pas d'un indicateur mais de méthodes d'analyses spécifiques construites sur des référentiels ad-hoc (Boulahbal, 2001). (iii) La troisième dimension est celle des coûts de la mobilité individuelle : les coûts temporels sont mesurés par le BTT (voir plus haut) et mis en regard de la vitesse globale de déplacement sur une journée (rapport du budget distance au budget temps). L'appréhension du coût monétaire est plus délicate (on n'achète pas une carte orange pour une journée ni même une voiture), mais possible avec des sources complémentaires. (iv) La quatrième dimension considère l'intensité d'usage des modes de transport par les individus, reflet de la compétitivité des modes du point de vue de l'individu. L'analyse est menée en termes de mode principal, en repérant pour chaque individu le mode qui a porté le plus long déplacement de sa 
journée, mais elle peut aussi être mené sur le chaînage des modes utilisés au cours de chaque sortie du domicile (nommée encore boucle de déplacement), ou de la journée (nombre et types de modes de transport utilisés, kilométrage et temps concernés par chaque mode). Le faible nombre d'analyses menées en termes de chaînages des modes relève plus de la rareté de la pratique que de la difficulté de son traitement. En Île-de-France 95\% des déplacements d'une personne qui a utilisé la voiture au moins une fois dans la journée sont réalisés en voiture, $95 \%$ des déplacements chaînant plusieurs modes concernent des chaînes de transport collectif et qui plus est des chaînes modales franciliennes. Ces chaînes comptent pour moins de $8 \%$ de la mobilité francilienne, pourcentage quasi stable depuis 25 ans. (v) La cinquième intègre dans l'analyse les capacités d'organisation des individus. Au premier chef sont caractérisés les accès au mode de transport : détention du permis de conduire, motorisation et " compétition " éventuelle entre adultes pour l'appropriation des véhicules, accès à l'offre de transport de public (qualité de la desserte en transport public du domicile et du lieu d'emploi, potentiels offerts par le réseau). Les capacités d'organisation des individus sont aussi mises en avant dans l'analyse des boucles de déplacements, qui assortie du taux de maximisation du chaînage (Boulahbal M., 1995), permet de mesurer comment les individus usent ou non de la mobilité pour " la contenir ". La mise en relation des performances des différents modes par les individus pour construire leur mobilité est appréciée en confrontant pour l'ensemble des boucles individuelles de déplacements, les temps et vitesse de déplacement associés à ces dernières par chaque mode (Massot MH, 2002). L'usage du téléphone en cours de déplacement, à une époque où les portables étaient encore peu répandus, révélait déjà une mise en œuvre de capacités d'organisation, surtout lors des schémas de mobilité les plus complexes (Massot MH., 1997). Les contraintes des individus dans l'accès individuel à l'emploi, mesurées par le nombre potentiel d'emplois accessibles par chacun à partir de sa localisation résidentielle pour un temps de transport donné, avec les moyens dont il dispose effectivement, sont qualifiables et qualifiés (Wenglenski S., 2003) et suggèrent d'importantes inégalités sociales dans ces potentiels, inégalités beaucoup moins visibles dans les pratiques elles mêmes.

Comme nombres d'analyses et notamment celles sur les modes de vie, l'analyse des pratiques de la mobilité et de leurs évolutions se construit par comparaison des comportements de groupes de population, ou par comparaison des mêmes groupes au fil du temps. Cette analyse procède donc par type d'individus qui préfigurent autant de contextes différents de production de la mobilité. Certains sont d'ordre démographiques comme le sexe, la position de l'individu dans le cycle de vie, la composition du ménage qui peut impliquer des rôles familiaux. D'autres sont d'ordre socioéconomiques (statut, profession, revenu, etc.). D'autres sont spatiaux comme la taille de l'agglomération de résidence, la localisation de la résidence par rapport aux pôles d'activités et aux dessertes intraurbaines et interurbaines. Ces contextes permettant de construire des typologies de population et de mobilité sont souvent présentés comme autant de déterminants structurels de la mobilité individuelle, agissant comme variable explicative de l'intensité de la mobilité. C'est peu discutable, à court comme à long terme pour la démographie. C'est acceptable à court terme pour le positionnement dans l'échelle sociale, moins à moyen terme : on sait par exemple que la durée du chômage est significativement influencée par la détention du permis de conduire et la conduite, toutes choses égales par ailleurs (Choffel P. \& Delattre E., 2003). Dans une perspective de long terme, le statut des localisations résidentielles est nettement plus incertain. À court terme le " Dis-moi où tu habites, je te dirai comment tu te déplaces " (Gallez C., Orfeuil JP., 1998) est un propos incontestable dans son sens causal. En revanche, sur la longue durée, les capacités des individus à se saisir des conditions de la mobilité (en termes de prix, de vitesse des modes et de 
structure des réseaux) étendent les univers de choix résidentiel, permettent d'intégrer d'autres critères que la proximité des principaux pôles d'activité (de la recherche de l'entre soi à celle de la nature en passant par la recherche de coûts acceptables pour le logement, (Pollachini AR. et Orfeuil JP., 1998) et transforment la localisation résidentielle en variable à expliquer, tout comme la mobilité quotidienne. Les conditions de mobilité transforment les potentiels de mobilité et les conditions d'arbitrage entre mobilité résidentielle et mobilité quotidienne. Ces deux types de mobilité s'avèrent ainsi partiellement substituables comme le sont reconnus plus largement l'agencement des fonctions urbaines et la mobilité quotidienne servant ensemble les besoins de co-présence des individus (Wiel M., 2002).

La mise en perspective de cet ensemble d'indicateurs qui font système a été réalisée, et c'est une ruse de l'histoire, à partir des enquêtes initiées dans les années soixante par les ingénieurs pour alimenter leurs modèles de trafic. Ces enquêtes sont aujourd'hui fréquemment mises au banc des accusés pour des raisons diverses, dont certaines sont recevables (elles ne portent le plus souvent que sur les pratiques très détaillées de déplacement d'un jour ouvrable alors même que les rythmes des modes de vie et de travail se diversifient). On remarquera cependant qu'elles ne sont guère différentes en structure de questionnement et de représentativité des enquêtes emploi du temps dont on valorise à juste titre les capacités heuristiques par ailleurs (Chenu A., 1998). Les travaux qui en résultent ont toujours répondu à un souci de validation/invalidation des concepts et des paradigmes. Les observations concernent pour l'essentiel le quart de siècle écoulé. Ils ont permis d'identifier des processus, d'éclairer de nombreux débats, et de renouveler le questionnement sur d'autres. Ils ont aussi réservé quelques surprises. Nous sélectionnons ci-dessous quelques résultats parmi les plus saillants.

La capacité de mouvement et la vitesse ont fait exploser l'exiguïté de la ville héritée... L'observation sur longue période des pratiques de mobilité valide le processus de co-construction de la ville et des potentiels de mobilité, via la quasi constance des budgets temps. Si l'on peut se plaire à discuter de telle ou telle évolution marginale du Budget-Temps de Transport ici ou là, on ne peut contester que nous parcourons au total une distance quatre fois plus grande qu'il y a 40 ans, et que cela ne s'est pas fait au prix d'une multiplication par quatre de nos durées de déplacements. En bref, la période a été marquée par la croissance des vitesses. Ce résultat, aujourd'hui largement accepté, a fait longtemps débat dans une période où les experts rejoignaient la perception sensible des citadins qui ne voyaient que la croissance des embouteillages. L'espace urbanisable s'est étendu (Les urbanistes nous rappellent que la "tache urbaine" ou la "consommation d'espace par habitant" ont doublé ou triplé en un quart de siècle), tout comme l'espace effectivement pratiqué (même par les résidents de la "ville héritée) s'est étendu. Le choix de nos espaces de pratiques d'activités comme celui de nos résidences s'est diversifié, les espaces les plus périphériques ont pu être conquis lorsque la multi-motorisation est devenue économiquement possible, mais nous consacrons approximativement le même budget temps à nos déplacements depuis que des enquêtes permettent cette mesure (enquêtes déplacements comme enquêtes emploi du temps). L'offre de vitesse conditionne la possibilité de s'approprier des espaces plus vastes, elle conditionne aussi nos possibilités d'installation à l'écart des flux et la possibilité pour les acteurs de redéployer leurs offres dans l'espace. En bref, la mobilité ne peut être appréhendée uniquement comme une résultante, la capacité de mobilité individuelle donne aussi à chacun un pouvoir organisateur sur l'espace, celui où il choisit de résider (du moins quand 
il a les capacités stratégiques de se placer sur les marchés immobiliers), celui où il choisit de réaliser ses activités.

L'observation globale reste vraie à un niveau plus désagrégé, par genre, situation sociale ou type de localisation. Il va sans dire que les enfants et les plus âgés d'entre nous n'ont pas le même budget temps que les adultes, ni la même intensité de déplacement (un actif qui travaille réalise trois fois plus de déplacements par jour qu'un retraité et parcourt presque quatre fois plus de kilomètres que ce dernier) mais chacun d'eux a le même budget temps de transport qu'hier. En outre, à un moment donné, les résidents des centres (qui parcourent des distances faibles) et les résidents des périphéries (qui parcourent des distances trois plus importantes) passent le même temps en déplacement. La seule véritable entorse à cette homogénéité se manifeste quand on compare l'Île-de-France (1h30) et la province (1h), l'entorse est importante, mais elle était de même ampleur en 1975 : la différence s'explique en partie par la taille exceptionnelle de l'Île-deFrance et beaucoup par les conditions d'usage des modes : l'Île-de-France est le seul territoire où les faibles capacités de stationnement de certains espaces contraignent à se reporter sur les modes plus lents que l'automobile. On observe d'ailleurs la même entorse dans les grandes villes de Suisse alémanique.

\section{Mais les moteurs principaux de la croissance sont derrière nous}

La validation de ce processus de croissance par l'offre de vitesse ne permet pas d'envisager pour l'avenir, pas plus d'ailleurs que pour le passé récent, une explosion de la mobilité. Si l'on admet en effet que les grands déterminants de l'extension de la sphère du quotidien sont l'accès à l'automobile, la vitesse, la diversité des territoires praticables par ce mode, et l'évolution favorable passée des coûts du transport, force est de constater que ces signaux forts passent progressivement du vert au rouge, du moins pour les mobilités urbaines ${ }^{3}$. Nous disposons aujourd'hui de 600 voitures pour mille habitants, l'équipement automobile des foyers est largement majoritaire dans toutes les catégories sociales, plus de $80 \%$ de la population adulte dispose du permis. Une part importante des familles avec enfants vit déjà dans les territoires dits périurbains. Ce qui est acquis n'est plus à l'être, les taux de croissance ne peuvent que s'infléchir fortement, et le phénomène s'observe déjà : depuis les années soixante-dix, le rythme de croissance des circulations est divisé par deux d'une décennie à la suivante ${ }^{4}$. Dans toutes les catégories sociales, les populations équipées sont majoritaires, les cadres supérieurs " hyper mobiles ", ceux dont la mobilité physique est la plus forte aussi bien en interaction sociale qu'en termes de distances parcourues sont ceux dont les mobilités urbaines croissent le moins et se stabilisent progressivement. Nous sommes ici à la fin d'un processus à l'œuvre depuis 50 ans, où les différentes populations ont eu progressivement et successivement accès à la norme de la mobilité rapide, flexible et ont intégré cette norme pour mettre en œuvre leur projet social. Ce processus ne concerne plus aujourd'hui que certains retraités (notamment des veuves) entrés tardivement dans le processus (ce sont ceux en effet dont la mobilité en termes d'interactions ou de distances parcourues a le plus augmenté récemment) et les plus pauvres ou précaires : le réservoir de croissance de la motorisation est devenu faible. Qu'en est-il du côté des usages ? La

\footnotetext{
3 Ce diagnostic ne s'applique pas à la mobilité interurbaine ou de longues distances, mobilité qui continue de croître fortement et qui par ailleurs ne relève pas au niveau individuel des mêmes processus de production que la mobilité plus récurrente et de courte distance qu'est la mobilité urbaine.

4 Il n'y a pas là de quoi prédire une croissance exponentielle de la mobilité (Vodoz et coll, 2004), du moins dans les pays européens fortement motorisés.
} 
production sociale de la vitesse est en panne pour l'automobile en ville : les nouvelles infrastructures sont difficiles à faire accepter aux riverains et donc très coûteuses, le patrimoine existant doit être partagé avec les piétons, les vélos, et les autobus, l'amour du tramway n'apportant, en matière de vitesse, que des progrès marginaux. Ces tendances ont un caractère durable, car les politiques qui les produisent se fondent sur une rationalité «en fins » exprimée par les citoyens à travers des thématiques comme celles de la pollution. On peut toutefois penser que la demande est plus profonde. Au-delà de la pollution, n'exprime-t-elle pas un malaise face à la société " hyper moderne ", " caractérisée par la sur mobilité et l'urgence et se traduisant pour quelques groupes sociaux dominants par une hyper activité dans le travail et le hors travail fonctionnant sur le mode de réactions rapides à des injonctions en continu " (Ascher F. 2002) ? N'y a-t-il pas là un plaidoyer pour quelque ralentissement et quelque recherche de stabilité dans un univers où les repères se délitent ? Enfin, du côté des coûts et des prix, les constructeurs continueront certes à faire des efforts (y compris avec la redécouverte de véhicules "populaires "), mais les probabilités de hausse des prix des carburants sont importantes, l'effet d'aubaine de la "diesélisation" s'épuise, et les transports publics pourraient faire plus appel à l'usager si l'on va vers des modes de gestion plus libérale.

\section{La mobilité débridée : un fantasme à tempérer ?}

La mobilité dans la société hyper moderne, «se débride, s'éparpille, la mobilité revendique, la mobilité décloisonne " et " les récurrences d'hier laissent place à une mobilité zigzagante, labile, tributaire d'un système de lieux dont le réseau se recompose en permanence et d'un système de temps sans cesse bouleversé et ....cristallise une révolution anthropologique et sociétale » (Marzloff B., 2004). La mobilité en question ici est plus large que celle nous analysons puisqu'elle rend compte de l'interpénétration de la mobilité virtuelle et physique dans nos modes de vie et de travail. Pour la mobilité physique quotidienne, les observations récentes n'attestent pas d'une telle complexification des schémas de mobilité, et nous ne sommes pas convaincus que cela soit dû au " retard abyssal des indicateurs et autres outils d'analyse pour la caractériser ", (Marzloff B., 2004). En effet, le nombre moyen de déplacements par personne et par jour est constant en Île-de-France depuis 25 ans. Résultat de la mise à la norme de mobilité (voir plus haut), les moins mobiles d'hier (les femmes et les retraités) se déplacent un peu plus et les actifs légèrement moins, si bien qu'on assiste à une concentration croissante de la population sur une plage de 2 à 4 déplacements par jour. La mobilité urbaine de loisirs, moins contrainte et plus diversifiée en destinations, progresse certes, mais les distances parcourues par l'ensemble de la population pour ce motif ne représentent encore qu'un quart des distances liées aux activités professionnelles pendant les jours ouvrables. Pas d'explosion de la mobilité depuis 25 ans, mais pas de complexification non plus des schémas de mobilité ou de croissance d'une" mobilité zigzagante" (Cerri V., 2004) : le nombre de sorties du domicile par jour et par personne a légèrement augmenté en Île-de-France depuis 25 ans, mais celles impliquant plus de deux déplacements stagnent alors que les sorties les plus simples, avec un aller retour au domicile sans autre déplacement augmentent. Ce phénomène est grandement partagé, on l'observe pour la majorité des catégories sociales et des situations résidentielles et sociales y compris donc chez les cadres supérieurs, ceux qui se singularisent encore par la mobilité la plus intense et la plus complexe (2.8 déplacements par sortie de domicile). Si hyper modernité il y a, il semble qu'au quotidien pour tous elle se réalise au domicile, et pour les actifs au domicile et sur le lieu de travail, lieu vers lequel les déplacements a eu tendance à croître cette dernière décennie. D'autres observations concernent l'ancrage territorial de la mobilité. L'observation statistique, sur une 
population globale, témoigne de la multiplication des lieux d'activité et d'un certain affaissement des centres. Au niveau de chaque individu en revanche, la mobilité s'organise autour de deux ou trois pôles : la commune de résidence, la commune centre, et le lieu de travail pour les actifs (Boulahbal M., 2001), le seul écart à cette " sobriété " territoriale étant portée par certains employés et ouvriers aux lieux de travail variables (5\% de la population, Crague G., 2000). Analysée sur la base des pratiques de mobilité individuelles recueillies sur un mois, la diversité des lieux pratiqués par les adultes franciliens n'apparaît pas plus importante : en moyenne sur un mois, l'adulte francilien ne pratique que 3 communes différentes y compris sa commune de résidence, l'étendue du spectre étant le suivant : $21 \%$ ne pratiquent que leur commune de résidence, $54 \%$ pratiquent 2 ou 3 communes et $25 \%$ quatre communes ou plus (Massot \&alii., 1998).

\section{Une rationalité par les fins qui a encore de beaux jours devant elle}

$\mathrm{Si}$, comme nous l'avons vu, la rationalité en valeur du citoyen est agissante sur la production des conditions de la mobilité, les pratiques semblent bien relever d'une action rationnelle «en finalité », (Weber M. ,1995), c'est-à-dire d'une action qui procède d'un ajustement des moyens aux fins. La vitesse joue un rôle central dans cette recherche d'efficacité individuelle. En effet, une écrasante majorité des conducteurs ( $92 \%$ des conducteurs franciliens et $85 \%$ des lyonnais) se déplaçant, un jour donné, ne pourraient pas réaliser leur programme d'activités dans les lieux où ils les ont réalisés avec un autre mode de transport (marche, vélo, transport collectif) à moins de consacrer plus de temps à se déplacer (Massot MH. \& alii, 2002). Le fait est majeur : au-delà de rendre compte du choix pour le mode de transport le plus rapide, cet ajustement du mode de transport aux programmes d'activités témoigne de la capacité des individus à intégrer la vitesse de déplacement comme principe d'organisation. Collectivement le fait est tout aussi majeur, puisque plus de $85 \%$ des trafics quotidiens sont portés par l'usage de la voiture et le défi de vitesse et de flexibilité que pose la voiture aux politiques "régulationnistes" est a priori très élevé. Dans la structure actuelle des modes de vie, de travail, d'organisation spatiales des activités et de l'habitat, et du coût de la mobilité pour l'individu, le potentiel maximal de report des mobilités «automobile» actuelles sur le transport public est en effet estimé au mieux à $10 \%$ des circulations, sous réserve d'une pénalisation de la vitesse de progression de la voiture et d'une hausse quantitative et qualitative considérable de la qualité de l'offre de transport public (Massot MH \& alii., 2003). Ces résultats augurent certes d'un certain scepticisme sur l'orientation et le discours des politiques " régulationnistes " actuels : si l'usage de la voiture doit être régulé et juste un peu plus que marginalement, comprenons ici que le report de ces mobilités vers le transport public sera faible. Plus profondément, ces résultats montrent que, dans un contexte de régulation de l'usage de la voiture, l'inversion de la dynamique actuelle de construction des mobilités quotidiennes urbaines sera quasi inévitable et supposera pour beaucoup d'entre nous un «resserrement des territoires » et - ou- une mobilisation d'autres techniques de communication interindividuelle.

\section{Des tensions qui ont vocation à perdurer}

Les éléments de panorama des pratiques brossés ci-dessus pourraient accréditer un sentiment d'absence de tensions réelles dans le champ. Ce n'est pas tout à fait le cas, et il faut alors rechercher du côté des différences de pratiques d'une part, du côté des écarts entre aptitudes individuelles à se déplacer, pratiques possibles et injonctions émises par une société fondée sur la mobilité individuelle pour ne pas dire autonome de l'autre. De grandes différences perdurent en 
effet, au-delà des grandes structures et structuration des pratiques de mobilités autour de la norme, entre les franges les moins mobiles et celles les plus mobiles. L'entrée par le revenu est ici un des révélateurs du poids des contraintes monétaires sur les pratiques de mobilité. En France, au sein du quart le plus modeste des ménages, seule la moitié des adultes disposent du permis de conduire, contre $90 \%$ pour ceux qui appartiennent au quart le plus aisé. Ces différences dans l'accès aux modes de transport conditionnent un usage des modes et des pratiques de déplacement radicalement différents. La marche est caractéristique du tiers des déplacements des populations modestes contre moins du quart pour la population totale ; et la voiture concerne moins de la moitié des déplacements des pauvres contre près des deux tiers en moyenne nationale. La moyenne quotidienne des distances parcourues s'élève à $15 \mathrm{~km}$ dans les populations les plus modestes, à $29 \mathrm{~km}$ chez les plus aisées, et les écarts sont encore beaucoup plus significatifs pour les pratiques à longue distance. Il y a là une source potentielle d'amertume, pour reprendre un terme désormais utilisé par des économistes (Abraham C. et Piron V., 2001). En outre, le revenu n'est qu'un indicateur des " capitaux " nécessaires à une pratique " sans souci " de la mobilité dans un espace aujourd'hui de plus en plus structuré par l'automobile. L'âge et les problèmes de santé et de motricité peuvent constituer des handicaps, et la part des personnes âgées est amenée à croître. La mobilité exige des capacités de programmation des activités, des compétences dans l'usage d'outils (cartes, automates, véhicules, etc.), une connaissance de la distribution des activités dans l'espace et des possibilités offertes par les réseaux qui résultent d'apprentissages qui n'ont pas toujours été accomplis par des franges précaires et aux parcours heurtés de la population (Le Breton E., 2003). Ce qui n'était que très partiellement un problème hier, où la fréquentation de territoires familiers et peuplés de familiers pouvait suffire à l'écoulement d'une vie, devient plus problématique aujourd'hui dans des territoires formatés par l'automobile, dans une société où les lieux d'activités sont éclatés.

Ceci nous introduit alors, de façon générale, à de nouvelles catégories et outils d'analyse qui ne reposent plus exclusivement sur les pratiques effectives de mobilité : la conquête joyeuse de la mobilité autonome n'est qu'une des facettes d'une médaille. L'autre est celle des systèmes d'injonction qui, partant des capacités générales de mobilité construites par les grandes infrastructures et la motorisation quasi généralisée, postulent une possibilité vite transformée en obligation. Les tensions se font jour lorsque l'écart est trop grand entre les injonctions et ce que les individus considèrent comme leurs possibilités réelles, qu'elles soient économiques, socioculturelles ou psychosociales. C'est le cas pour les plus démunis qui doivent aujourd'hui se déplacer sur des «terra incognito » ou par le biais de systèmes techniques dont l'usage demande un apprentissage qu'ils n'ont pas eu; c'est le cas pour d'autres qui, refusant de quitter des territoires où sont leurs liens familiaux et sociaux, ne peuvent suivre la délocalisation d'un site de production. C'est le cas pour d'autres encore, ancrés dans le système productif actuel qui requiert une flexibilité et une réactivité importante pour satisfaire un client toujours plus exigeant sur la qualité et les délais, si les conditions de congestion des réseaux ne permettent pas de réaliser les programmes d'activité complexes sous-tendus par le système ou qu'ils ne le peuvent qu'au prix de conditions de déplacements difficiles. L'importance prise par la mobilité dans les sociétés contemporaines, c'est aussi et surtout cela : une norme et une valeur sociale produites par différents secteurs de la société, des hypermarchés à l'Agence Nationale Pour l'Emploi, face auxquelles nous sommes plus ou moins en situation de nous conformer mais qui en retour modifient subrepticement nos modes de vie. 
Autant dire que l'explosion passée de la mobilité n'est pas prête d'épuiser les débats, et que les débats sur le statut de la mobilité et ses «formes» futures noirciront nombre de pages, les évolutions qualifiant notre société et notre système de production, qu'ils soient hyper modernes ou non, n'étant pas encore toutes analysées. Ces débats, portant sur un objet aussi complexe que la mobilité individuelle, se doivent, dans un contexte de plus en pluridisciplinaire, d'être fondés sur des concepts plus nombreux et très précis, se référant à des contextes individuels et sociaux non moins précis, et des cadres interprétatifs explicites.

\section{Références bibliographiques}

ABRAHAM C., PIRON V., 2001, Amertume et acceptabilité des péages, in Cahiers Scientifiques du Transport $n^{\circ} 40$.

ASCHER François, 2000, Ces évènements nous dépassent, feignons d'en être les organisateurs, Essai sur la société contemporaine, Éditions de l'aube, page 202

AUBERT Nicole (Dir), 2004, L'individu hyper moderne, Éditions Eres

BAUMAN Zygmunt, 2000 Liquid modernity, Polity press, Cambridge, U.K.

BONVALET Catherine, MASON Dominique, 1999, Famille et entourage : le jeu des proximités, in La famille et ses proches. L'aménagement des territoires, INED-PUF.

BOULAHBAL Mouna, 1995, Le chaînage des déplacements : interface entre activité et mobilité individuelle Mémoire de DEA, ENPC-Université de Paris 12. Direction JP. Orfeuil

BOULAHBAL Mouna, 2001, Effet polarisant du lieu de travail sur le territoire de la vie quotidienne des actifs », in Recherche Transport Sécurité, n73pp.43-63

BOVY P., SALOMON I., ORFEUIL J.P, 1993, A billion trips a day : tradition and transition in European mobility

CASTEL Robert, 1995, Les métamorphoses de la question sociale, Fayard

CERRI Valérie, 2004, Diversité et complexité de la mobilité des déplacements en Île-De-France, DEA Transport, Université Paris XII, sous la direction de JP. Orfeuil.

CERTU, (2002), L mobilité urbaine en France : les années 90, Éditions de CERTU, Collection Références n 26

CHENU Alain, 2002, Les horaires et l'organisation du temps de travail ", in Economie et Statistiques, INSEE, n³52353

CHOFFEL P. et DELATTRE E., 2003 " Habiter un quartier défavorisé : quels effets sur la durée du chômage ? Premières synthèses $n^{\circ} 43.1$, Dares, Octobre

DURKHEIM Emile, 1894, Les règles de la méthode sociologique, Flammarion

EHRENBERG Alain, 1996, L'individu incertain, Hachette Pluriel

GOODWIN Peter, KITAMURA R., 1989, Some principles of dynamic analysis of travel behaviour in Developments od dy,namic analysis and activity based approaches, Jones P. Ed, Gower

CRAGUE Gilles, 2000, Economie d'agglomération : de la spécialisation à la commutation, Thèse de Doctorat ENPCLATTS

GALLEZ Caroline., ORFEUIL Jean-Pierre, 1998, «Dis-moi où tu habites, je te dirai comment tu te déplaces », in PUMAIN Denise et MATTEI Marie-Françoise (Ed.), Données urbaines, ${ }^{\circ} 2$, Anthropos, mai 1998, pp.157-164

HÄGERSTRAND Torsten, 1970, "What about people in the regional science ?", in Papers in regional Science associaltion, $\mathrm{n}^{\circ} 24, \mathrm{pp} 7-21$

HÄGERSTRAND Torsten, 1987, Human interaction and spatial mobility: retrospect and prospect, in Transportation planning in a changing world, Nijkamp P. et Reichmann S. (eds.) Aldershot - Grower, pp. 11-27

KENWORTHY J., LAUBE F., 1999, An international sourcebook on automobile dependence in citiies 1960-1990, University press of Colorado 
LE BRETON Eric., 2004 Exclusion et immobilité : la figure de l'insulaire, in Orfeuil, 2004 (Dir) Transports, pauvretés exclusions, éditions de l'Aube

LIPOVETSKI Gilles, 1983, L'ère du vide, Gallimard -Folio Essais

MARZLOFF Bruno, 2004, On a ouvert la boîte de Pandore de la mobilité, in Mobilités. Net, Villes Transports, technologies face aux nouvelles technologies, Éditions L.G.D.J. Questions numériques

MASSOT Marie-Hélène., ARMOOGUM Jimmy., 2002, Évaluation des potentiels de report modal des trafics automobiles dans le cas de la zone dense francilienne, in Revue Transport Sécurité, RTS 77, pp. 250-280.

MASSOT Marie-Hélène \& alii, 1997, Automobile et transports publics : complémentarité des pratiques modales dans l'aire d'influence de la RATP, in Entreprendre la ville : nouvelles temporalités, nouveaux services, coordonné par Obadia Alain, Éditions de l'aube, Collection Territoires

MASSOT Marie-Hélène 1997, L'usage du téléphone en cours de déplacement, in Revue Transport Sécurité n 56

MERLIN Pierre, 1998, Les mobilités urbaines :données et analyses, in Données urbaines n², eds Anthropos, Collection Villes, pp 139-145

NEWMAN P. KENWORTHY J., 1989, Cities and automobile dependence, Gower

POLLACHINI Anna Rita, ORFEUIL Jean-Pierre, Les dépenses des ménages franciliens pour le logement et les transports, in Revue Transport Sécurité $n^{\circ} 63$

ORFEUIL Jean-Pierre, 2000, L'évolution de la mobilité quotidienne : comprendre les dynamiques, éclairer les controverses, Synthèse INRETS n ${ }^{\circ}$, Les collections de l'INRETS

ORFEUIL Jean-Pierre (dir.), 2004, Transports, pauvretés, exclusions. Pouvoir bouger pour s'en sortir, Editions de l'Aube, Paris, 2004, 181p

SHAFER A. ET VICTOR D.G., 2000, The future mobility of the world population, Transportation Research A, $\mathrm{n}^{\circ}$ 34-3, April

PUCA, 2003, Mobilité et territoires urbains, PUCA, Paris

SOROKIN P.A., 1927, Social Mobility, Harper and brothers, NY, 1927

SZALAÎ (1972), The use of time : daily activities of urban and suburban populations in twelve countries, Mouton.

VODOZ Luc, PFISTER GIAUQUE B. et JEMELIN Christophe, 2004, L'aire du temps, Presses polytechnique et universitaires romandes

VULPIAN Alain, 2004, À l'écoute des gens ordinaires, Dunod

VOORHES Alan M., 1959, " Land use and traffic models ", Journal of the American Institute of planners n²5

WEBER Max (1995), Économie et Société, réédition, Press Pocket

WIEL Marc, 2002, Ville et automobile, Editions Descartes et Cie, Paris

ZAHAVI Yacob, 1974, Travel time budgets and mobility in urban areas, Report for the U.S. Department of Transportation, Washington, D.C. 